\title{
ANTIMICROBIAL RESISTANCE IN COAGULASE-NEGATIVE STAPHYLOCOCCI
}

\author{
J. M. T. Hamilton-Miller and A. Iliffe
}

Department of Medical Microbiology, Royal Free Hospital School of Medicine, Pond Street, London NW3

\begin{abstract}
Summary. Patterns of resistance to antimicrobial agents were studied in 193 strains of coagulase-negative staphylococci isolated from hospital patients. Strains isolated from patients with malignant disease were significantly more often resistant to sulphonamide, trimethoprim, gentamicin and methicillin than were strains from other sources. Susceptibility to various $\beta$-lactam antibiotics and aminoglycosides was investigated in members of the two most frequent species: Staphylococcus epidermidis and $S$. haemolyticus. S. haemolyticus strains were not only more often resistant to methicillin than $S$. epidermidis strains (respectively $81 \%$ and $17 \%$ ) but they were more highly resistant (mean MICs respectively 85 and $19 \mathrm{mg} / \mathrm{L}$ ). Methicillin-resistant $S$. haemolyticus strains were highly resistant to nine other $\beta$-lactam antibiotics, whereas methicillin-resistant $S$. epidermidis strains showed both lower levels and a narrower spectrum of cross-resistance. Resistance to methicillin in members of both species was "heterogeneous", i.e., only a minority of cells in a culture showed significant resistance. Almost all gentamicin-resistant strains were sensitive to netilmicin and amikacin; rifampicin, vancomycin and teicoplanin were also highly active in vitro.
\end{abstract}

\section{INTRODUCTION}

Recent changes in therapeutic practice, especially those that have led to the longer survival of patients with defective immunity and the increased use of intravenous catheters, have given a new importance to coagulase-negative staphylococci (CNS) as pathogens (Lowy and Hammer, 1983). If the presence of CNS in a specimen is judged to be clinically significant, it may be difficult to decide upon appropriate antibiotic therapy because the use of two important groups of antibiotics - the $\beta$-lactams and the aminoglycosides - may be ruled out by the presence of resistance to them (Archer and Tenenbaum, 1980; Richardson and Marples, 1982; Karchmer, Archer and Dismukes, 1983).

To clarify several outstanding questions in relation to drug resistance among CNS, we surveyed resistance patterns in organisms isolated from a highly "at risk" population-patients undergoing cytotoxic treatment for various leukaemias - and compared these with strains isolated from other types of patients. 


\section{MATERIALS AND METHODS}

\section{Antibiotics}

The following compounds were obtained from the manufacturers: methicillin, flucloxacillin, cloxacillin (Beecham Research Laboratories); streptomycin, cephalexin, ceftazidime (Glaxo Laboratories Ltd); tobramycin, cephalothin, cefamandole, vancomycin (Eli Lilly and Co. Ltd); gentamicin, cefotaxime (Roussell Laboratories Ltd); teicoplanin, rifampicin (Merrell Pharmaceuticals Ltd); netilmicin (Schering Pharmaceuticals); kanamycin (Bristol-Myers Pharmaceuticals); neomycin (Biorex); cefoxitin (Merck Sharp and Dohme Ltd); and cephradine (E.R. Squibb and Sons Ltd).

\section{Bacterial strains}

Strains of CNS (193 in number), isolated between November 1982 and November 1983, were investigated; 132 of them were from surveillance cultures (mostly nose and throat swabs) from patients being treated for a haematological malignancy (most commonly acute myeloid leukaemia); 61 strains were from patients without malignant disease, most of whom had in-dwelling intravascular lines of one kind or another.

The methods of Marples (1981) and Marples and Richardson (1982) were applied to identify 182 of these strains; $126(69 \%)$ were allocated to biotype SII and $32(18 \%)$ to biotype SVIh. These correspond to Staphylococcus epidermidis and S. haemolyticus (Kloos and Schleifer, 1975) respectively (Marples, 1981). Of the remaining strains, $11(6 \%)$ belonged to biotype SIII, two to biotype SV, and 11 were not identifiable. Full details of these strains are given in a separate paper (Hamilton-Miller and Iliffe, in press).

\section{Sensitivity testing}

Disk test. IsoSensitest Agar (Oxoid) was inoculated with $c .10^{4} \mathrm{cfu}$ of staphylococci per plate, and set with disks containing the following antibiotics $(\mu \mathrm{g})$ : clindamycin (2), erythromycin (5), fusidic acid (10), gentamicin (10), rifampicin (5), Sulphatriad (200), tetracycline (10), trimethoprim $(2 \cdot 5)$. Plates were incubated at $37^{\circ} \mathrm{C}$ overnight. Some of the gentamicin-resistant strains were further tested against seven other aminoglycosides: amikacin (10), kanamycin (30), neomycin (10), netilmicin (10), sissomicin (10), streptomycin (10) and tobramycin (10). Interpretation of "resistance" in terms of zone diameters was as laid down in the manufacturer's specifications (Oxoid, 1980; and package inserts).

Strip test. Six strains were tested on each 85 -mm-diameter plate of Oxoid Nutrient Agar. They were streaked in equidistant parallel lines by means of a wool swab that had been dipped in a bacterial suspension containing $c .10^{6} \mathrm{cfu} / \mathrm{ml}$. A paper strip $(57 \times 6 \mathrm{~mm})$ containing $25 \mu \mathrm{g}$ of methicillin (Mast Laboratories, Liverpool) was laid at right angles to the streaks. The plates were incubated at $30^{\circ} \mathrm{C}$ overnight. The Oxford staphylococcus (NCTC 6571), which was included on every plate, consistently gave a zone of between 10 and $11 \mathrm{~mm}$ on each side of the strip. Most of the resistant strains grew right up to the strip, but any strain showing a markedly decreased zone (i.e. $5 \mathrm{~mm}$ or less) was also recorded as resistant. The majority of strains were also tested in parallel at $37^{\circ} \mathrm{C}$.

Determination of MIC. A large inoculum was used, in order to detect heterogeneous resistance (Jones et al., 1983). Each strain was shaken $(100 \mathrm{rpm})$ at $37^{\circ} \mathrm{C}$ overnight in $10 \mathrm{ml}$ of IsoSensitest Broth (Oxoid) in a 100-ml conical flask in an orbital incubator (Gallenkamp); $0 \cdot 7 \mu \mathrm{l}$ $\left(c .2 \times 10^{7} \mathrm{cfu}\right)$ was inoculated on to plates of IsoSensitest Agar containing doubling dilutions of 19 antibiotics. Plates were examined after overnight incubation at $37^{\circ} \mathrm{C}$, the MIC being taken as the lowest concentration that allowed the growth of 10 colonies or fewer.

Population structure in respect of methicillin resistance. Organisms were grown overnight in Digest Broth (Southern Group Laboratories) and $0 \cdot 1-\mathrm{ml}$ volumes of decimal dilutions (to $10^{-7}$ ) were spread on to a series of plates of IsoSensitest Agar containing doubling dilutions of methicillin $(0.5$ to $1024 \mathrm{mg} / \mathrm{L})$. Plates were incubated at $37^{\circ} \mathrm{C}$ and the proportion of the population able to grow at each antibiotic concentration was calculated. For one strain $(S$. haemolyticus no. 640) two extra series of plates were prepared and were incubated at $40^{\circ} \mathrm{C}$ and $28^{\circ} \mathrm{C}$ respectively. 
Statistical analysis

Differences in the occurrence of resistant strains from different sources were checked for significance by the $\chi^{2}$ test, with Yates's correction applied when necessary. For analysis of MICs for organisms of various sensitivity classes (viz. tables II and III), Student's $t$ test was applied to logarithms of the individual MIC results.

\section{RESULTS}

Antibiotic resistance in strains isolated from patients with malignant disease and from other patients

The results of disk tests with nine antibiotics are shown in table I. There was no significant difference in the frequency of resistance to clindamycin, rifampicin or tetracycline between the two groups of organisms. However, resistance to sulphonamide, trimethoprim, gentamicin and methicillin was significantly more common in patients with malignancy $(p<0.05)$. An unexpected finding was that organisms from patients with a non-malignant condition were more likely to be resistant to erythromycin and to fusidic acid than were organisms from patients with a malignancy $(\mathrm{p}<0.01)$.

Most of the gentamicin-resistant strains (90/101) were tested further against a wider range of aminoglycosides. Netilmicin was active against all but one strain, and only five were resistant to amikacin. There was complete cross-resistance between gentamicin and kanamycin, while tobramycin and sissomicin were each active against six strains only $(93 \%$ resistance). Thirty-one strains $(34.5 \%)$ were sensitive to neomycin and $52(58 \%)$ to streptomycin.

There was no difference in the frequency of resistance to individual aminoglycosides in gentamicin-resistant strains isolated from patients with a malignancy in comparison with strains from other patients, except in the case of streptomycin; $64 \%$ of the former $(47 / 73)$ but only $29 \%$ of the latter $(5 / 17)$ were sensitive to this antibiotic $(\mathrm{p}<0 \cdot 05)$.

Variation in resistance between staphylococcal species. $S$. haemolyticus strains were more often resistant $(\mathrm{p}<0 \cdot 01)$ than $S$. epidermidis strains to gentamicin (respectively $22 / 32$ and $52 / 125)$, to methicillin (26/32 and $21 / 125)$ and to both antibiotics $(21 / 32$ and $10 / 125)$.

\section{Methicillin- and gentamicin-resistant strains of S. epidermidis and S. haemolyticus}

Nine strains of each of these two species were selected for further tests of susceptibility to antibiotics.

MICs of $\beta$-lactam antibiotics. The 18 strains, and nine strains of methicillin-sensitive $S$. epidermidis, were tested against $10 \beta$-lactam antibiotics (table II). Two points emerged from the results. First, methicillin-resistant strains of $S$. haemolyticus were more resistant to all of these agents than were methicillin-resistant strains of $S$. epidermidis; geometric mean MIC values were significantly higher $(\mathrm{p}<0.05)$ in the former than the latter. Second, geometric mean MICs of other $\beta$-lactam antibiotics for methicillin-resistant $S$. epidermidis were in several cases not greatly in excess of the values found for methicillin-sensitive strains of the same species. The difference 


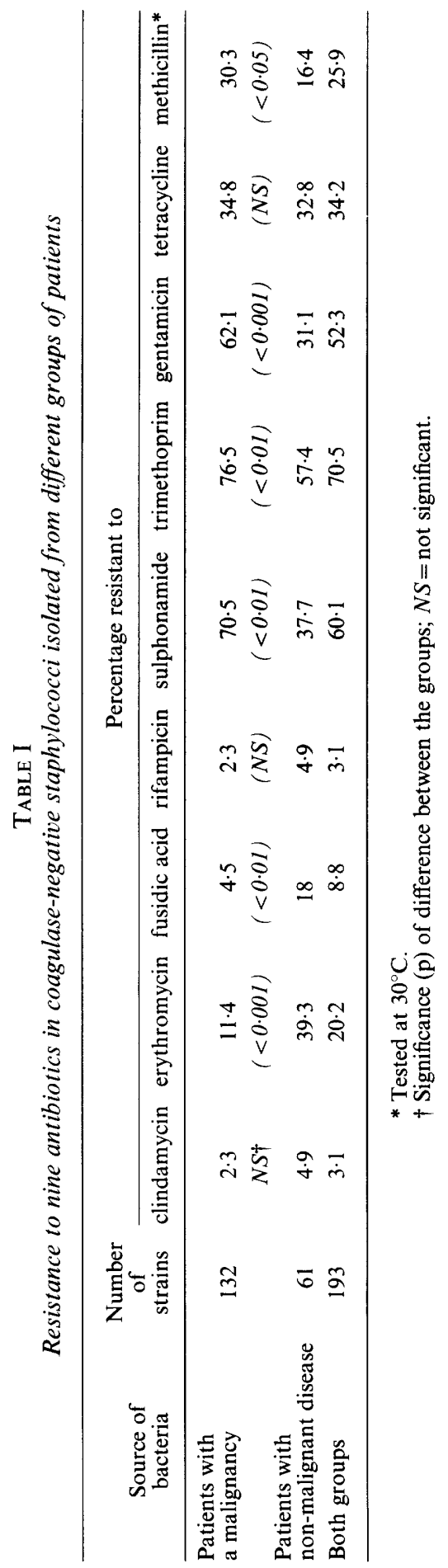


TABLE II

MICs of $\beta$-lactam antibiotics for nine strains each of methicillin-sensitive $S$. epidermidis, methicillin-resistant $S$. epidermidis and methicillin-resistant $S$. haemolyticus

\begin{tabular}{l|rrrr}
\hline & \multicolumn{3}{|c}{ Geometric mean MIC (mg/L) (and range) for } \\
\cline { 2 - 5 } Antibiotic & $\begin{array}{c}\text { methicillin- } \\
\text { sensitive } \\
\text { S. epidermidis }\end{array}$ & $\begin{array}{c}\text { methicillin- } \\
\text { resistant } \\
\text { S. epidermidis* }\end{array}$ & $\begin{array}{c}\text { methicillin- } \\
\text { resistant } \\
\text { S. haemolyticus* }\end{array}$ \\
\hline Methicillin & $3 \cdot 4(2-4)$ & $19 \cdot 1(1-128)$ & $85(16-1024)$ \\
Flucloxacillin & $0 \cdot 8(0 \cdot 25-2)$ & $3 \cdot 2(1-128)$ & $20(2-512)$ \\
Cloxacillin & $0 \cdot 9(0 \cdot 25-2)$ & $3 \cdot 2(1-128)$ & $40(4-512)$ \\
Cefamandole & $2 \cdot 3(0 \cdot 5-4)$ & $4 \cdot 6(2-16)$ & $20(16-32)$ \\
Ceftazidime & $11 \cdot 7(8-16)$ & 17 & $(8-128)$ & $130(32-256)$ \\
Cefotaxime & 4 & $(0 \cdot 5-8)$ & $7 \cdot 3(2-512)$ & $190(32-1024)$ \\
Cephalexin & $8 \cdot 6(0 \cdot 5-32)$ & $29 \cdot 5(8-256)$ & $305(128-512)$ \\
Cephradine & $8 \cdot 6(2-32)$ & $15 \cdot 9(4-64)$ & $200(128-256)$ \\
Cephalothin & $0 \cdot 8(0 \cdot 25-1)$ & $2 \cdot 1(0 \cdot 5-16)$ & $45(4-256)$ \\
Cefoxitin & $5 \cdot 4(2-16)$ & 17 & $(8-128)$ & $70(32-256)$ \\
\hline
\end{tabular}

* The strains were also gentamicin resistant (see table III).

TABLE III

MICs of aminoglycosides for nine strains each of gentamicin-resistant $S$. epidermidis and $S$. haemolyticus, and for the Oxford staphylococcus

\begin{tabular}{l|ccc}
\hline & \multicolumn{3}{|c}{ Geometric mean MIC (mg/L) for } \\
\cline { 2 - 4 } Antibiotic & S. epidermidis* & S. haemolyticus* & $\begin{array}{c}\text { Oxford } \\
\text { staphylococcus }\end{array}$ \\
\hline Neomycin & $31 \cdot 6$ & $7 \cdot 2$ & 1 \\
Streptomycin & 17 & $>512$ & 8 \\
Tobramycin & $25 \cdot 1$ & $46 \cdot 8$ & $0 \cdot 5$ \\
Netilmicin & 3 & $2 \cdot 3$ & $<1$ \\
Gentamicin & $23 \cdot 4$ & $33 \cdot 9$ & $<1$ \\
Kanamycin & $>512$ & $>512$ & 2 \\
\hline
\end{tabular}

* These strains were also methicillin resistant (see table II).

reached the formal level of statistical significance $(p<0.05)$ in respect of flucloxacillin, cloxacillin, cephalexin, cephalothin and cefoxitin but not of cefamandole, ceftazidime, cefotaxime and cephradine. With the latter four antibiotics, however, a small minority of strains showed a degree of resistance exceeding that of any of the methicillin-sensitive strains of $S$. epidermidis.

MICs of aminoglycosides. There were few clinically important differences between the geometric-mean MICs of individual aminoglycosides for members of the two species (table III). Their susceptibility to tobramycin, netilmicin, gentamicin and kanamycin was similar, though $S$. epidermidis strains were on average significantly more resistant to neomycin and less to streptomycin than S. haemolyticus strains.

MICs of other antibiotics. All the strains tested were sensitive to rifampicin, vancomycin and teicoplanin $(\mathrm{MIC}<1 \mathrm{mg} / \mathrm{L})$. 


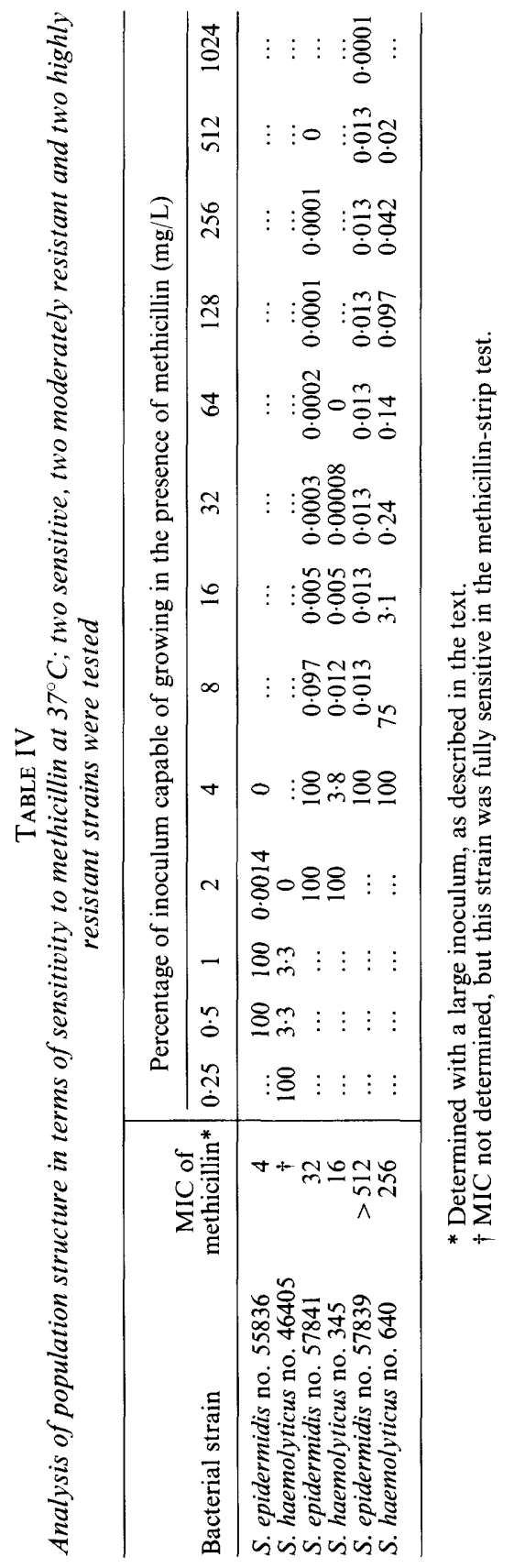




\section{Character of methicillin resistance in $C N S$}

Effect of temperature on the methicillin-sensitivity test. Of the 173 strains tested against methicillin at $30^{\circ}$ and at $37^{\circ} \mathrm{C}, 116(67 \%)$ were sensitive at both temperatures and $46(26.5 \%)$ were resistant at both temperatures; six were sensitive at $30^{\circ}$ but resistant at $37^{\circ} \mathrm{C}$ and five were resistant at $30^{\circ}$ but sensitive at $37^{\circ} \mathrm{C}$. It thus appears that methicillin resistance in CNS can be detected equally well at either temperature, in contrast to $S$. aureus, in which testing must be carried out at $30^{\circ} \mathrm{C}$ (Annear, 1968).

Heterogeneity of methicillin resistance. Six strains of CNS were submitted to population-structure analysis - one sensitive, one moderately resistant and one highly resistant representative each of S. epidermidis (nos. 55836, 57841 and 57839 respectively) and S. haemolyticus (nos. 46405, 345 and 640 respectively). The results of this experiment (table IV) demonstrated that methicillin-resistant strains of CNS contained only a minority of cells capable of growing in the presence of high concentrations of the antibiotic at $37^{\circ} \mathrm{C}$. The test was repeated with $S$. haemolyticus strain 640 at $40^{\circ}$ and $28^{\circ} \mathrm{C}$ (table V). Resistance was expressed most strongly at the lowest temperature and least at the highest.

TABLE V

Heterogeneity of resistance to methicillin in S. haemolyticus strain 640 at various growth temperatures

\begin{tabular}{l|ccc}
\hline & \multicolumn{3}{|c}{$\begin{array}{c}\text { Percentage of inoculum capable of growing at the } \\
\text { indicated temperature in the presence of methicillin }(\mathrm{mg} / \mathrm{L})\end{array}$} \\
\cline { 2 - 4 } $\begin{array}{c}\text { Growth } \\
\text { temperature }\left({ }^{\circ} \mathrm{C}\right)\end{array}$ & 4 & 32 & 256 \\
\hline 40 & $1 \cdot 9$ & $0 \cdot 008$ & $0 \cdot 00006$ \\
37 & 100 & $0 \cdot 25$ & $0 \cdot 042$ \\
28 & 95 & 39 & $0 \cdot 28$ \\
\hline
\end{tabular}

\section{DisCUSSION}

Study of the literature shows that there is still marked disagreement as to whether or not methicillin-resistant CNS show cross-resistance to the cephalosporins. On the one hand some workers (e.g., Lowy and Hammer, 1983; Karchmer et al., 1983) state that for clinical purposes methicillin-resistant CNS should be regarded as resistant to all cephalosporins irrespective of results of laboratory tests: this is also the case with methicillin-resistant $S$. aureus (Jones et al., 1983). On the other hand, there have been several reports that methicillin-resistant CNS are sensitive to certain cephalosporins in vitro (Marsik and Brake, 1982; Price and Flournoy, 1982; Gill et al., 1983; Winston et al., 1983), and cephalosporins have actually been recommended for treatment of infections with methicillin-resistant CNS (Archer, 1978; Winston et al., 1983). These differences may in part be attributed to the methods used by some of the investigators; for example, Lowy et al. (1983) showed that the broth-dilution method may fail to detect some resistant strains. However, many previous workers (e.g., Lowy and Hammer, 1983; Winston et al., 1983) did not speciate the CNS strains they tested, and our results show clearly that differences in the "mix" of S. epidermidis (sensu stricto) and $S$. haemolyticus would greatly affect the results obtained. We found that 
methicillin-resistant strains of $S$. haemolyticus showed markedly increased resistance to all seven cephalosporins tested with a large inoculum. On the other hand, methicillin-resistant strains of S. epidermidis (Marples biotype SII) had significantly raised mean levels of resistance to only some $\beta$-lactam antibiotics. Cephradine, cefamandole, ceftazidime and cefotaxime were almost as active against the majority of methicillin-resistant strains as against methicillin-sensitive strains.

Methicillin resistance in CNS, unlike that in $S$. aureus, can be detected as well at $37^{\circ}$ as at $30^{\circ} \mathrm{C}$ if a large inoculum is employed. Wilkinson et al. (1980) reported this for $S$. epidermidis, and our findings confirm their observations and extend them to $S$. haemolyticus. Analysis of population structure showed that methicillin resistance in $S$. epidermidis and $S$. haemolyticus was heterogeneous. In $S$. epidermidis it has been shown to be temperature-dependent (see, for example, Sabath, 1977, and Hansen, 1983); this was also so in our $S$. haemolyticus strain 640 . In these respects, therefore, methicillin resistance in the two species of CNS resembles that in S. aureus (Annear, 1968; Dyke, 1969).

In all, we found that $26 \%$ of the CNS we isolated were resistant to methicillin and $52 \%$ to gentamicin. These frequencies were greater in strains isolated from patients with malignant disease than in other patients. This is of potential importance, because patients under treatment for malignant diseases may well need antibiotics for generalised infections caused by CNS. Many of them have already received several antibiotics, including gentamicin and $\beta$-lactams, which tend to select resistant organisms. The increased resistance to trimethoprim and sulphonamide in our patients with malignant disease may be explained by the frequent use of prophylactic co-trimoxazole.

The pattern of resistance to aminoglycosides strongly suggested the presence in resistant strains of the inactivating enzyme aminoglycoside phosphotransferase (APT) $2^{\prime \prime}$, which is not active against netilmicin. Aminoglycoside acetylase $6^{\prime}$ always accompanies APT $2^{\prime \prime}$ in staphylococci (Shannon and Phillips, 1982) and if present in large amount causes resistance to amikacin. This probably explains the $5 \%$ of gentamicin-resistant strains which were found to be resistant to amikacin. Aminoglycoside resistance is plasmid-mediated in CNS; it has been reported recently that the plasmids can be passed from CNS to $S$. aureus, probably by conjugation as well as by transduction (Cohen et al., 1982; Jaffe et al., 1982; Forbes and Schaberg, 1983; McDonnell et al., 1983). Weinstein et al. (1982) consider that multi-resistant CNS strains may thus act as a reservoir of resistance for $S$. aureus.

Besides netilmicin and amikacin, the only antibiotics tested to which resistance was infrequent or not detected were rifampicin, vancomycin and teicoplanin. Brumfitt et al. (1983) found that the combinations rifampicin plus novobiocin and rifampicin plus teicoplanin had promising in-vitro action against multi-resistant staphylococci. We feel that future strategies for the treatment of infections caused by such organisms will depend upon the use of antibiotics at present considered to be in the "reserve" category.

This study was funded by a grant from The Royal Free Hospital Endowment Fund for which we are most grateful. We acknowledge the valuable help of Dr R.R. Marples in confirming the identity of some strains. 


\section{REFERENCES}

Annear D I 1968 The effect of temperature on resistance of Staphylococcus aureus to methicillin and some other antibiotics. Medical Journal of Australia 1:444-446.

Archer G L 1978 Antimicrobial susceptibility and selection of resistance among Staphylococcus epidermidis isolates recovered from patients with infections of indwelling foreign devices. Antimicrobial Agents and Chemotherapy 14:353-359.

Archer G L, Tenenbaum M J 1980 Antibiotic-resistant Staphylococcus epidermidis in patients undergoing cardiac surgery. Antimicrobial Agents and Chemotherapy 17:269-272.

Brumfitt W, Dixson S, Hamilton-Miller J M T 1983 Sensitivity of Staphylococcus aureus resistant to methicillin and gentamicin, with special reference to rifampicin. Proceedings of the 13th International Congress of Chemotherapy 83:1-4.

Cohen M L, Wong E S, Falkow S 1982 Common R-plasmids in Staphylococcus aureus and Staphylococcus epidermidis during a nosocomial Staphylococcus aureus outbreak. Antimicrobial Agents and Chemotherapy 21:210-215.

Dyke K G H 1969 Penicillinase production and intrinsic resistance to penicillins in methicillin-resistant cultures of Staphylococcus aureus. Journal of Medical Microbiology 2:261-278.

Forbes B A, Schaberg D R 1983 Transfer of resistance plasmids from Staphylococcus epidermidis to Staphylococcus aureus: evidence for conjugative exchange of resistance. Journal of Bacteriology 153:627-634.

Gill V J, Selepak S T, Williams E C 1983 Species identification and antibiotic susceptibilities of coagulase-negative staphylococci isolated from clinical specimens. Journal of Clinical Microbiology 18:1314-1319.

Hamilton-Miller $\mathbf{J}$ M T, Iliffe A Characterization of coagulase-negative staphylococci from patients with a malignancy compared with those from other sources. Zentralblatt für Bakteriologie Mikrobiologie und Hygiene Abt I, Orig A, (in press).

Hansen B G 1983 Population analysis of susceptibility to methicillin, vancomycin, and three cephalosporins in two methicillin-resistant strains of Staphylococcus epidermidis. Acta Pathologica, Microbiologica et Immunologica Scandinavica B91:279-284.

Jaffe H W, Sweeney H M, Weinstein R A, Kabins S A, Nathan C, Cohen S 1982 Structural and phenotypic varieties of gentamicin resistance plasmids in hospital strains of Staphylococcus aureus and coagulase-negative staphylococci. Antimicrobial Agents and Chemotherapy 21:773-779.

Jones R N, Edson D C, the CAP Microbiology Resource Committee 1983 Special topics in antimicrobial susceptibility testing: test accuracy against methicillin-resistant Staphylococcus aureus, pneumococci and the sensitivity of $\beta$-lactamase methods. American Journal of Clinical Pathology 80 Suppl:609-614.

Karchmer A W, Archer G L, Dismukes W E 1983 Staphylococcus epidermidis causing prosthetic valve endocarditis: microbiologic and clinical observations as guides to therapy. Annals of Internal Medicine 98:447-455.

Kloos W E, Schleifer K H 1975 Simplified scheme for routine identification of human Staphylococcus species. Journal of clinical Microbiology 1:82-88.

Lowy F D, Chang D S, Aning V, Williams S, Szilagyi G 1983 Reliability of in vitro susceptibility tests for detecting coagulase-negative staphylococcal resistance to penicillinase-resistant semi-synthetic penicillins. Journal of clinical Microbiology 18:1122-1126.

Lowy F D, Hammer S M 1983 Staphylococcus epidermidis infections. Annals of Internal Medicine 99:834-839.

McDonnell R W, Sweeney H M, Cohen S 1983 Conjugational transfer of gentamicin-resistance plasmids intra- and interspecifically in Staphylococcus aureus and Staphylococcus epidermidis. Antimicrobial Agents and Chemotherapy 23:151-160.

Marples R R 1981 Taxonomic studies of staphylococci and micrococci. In: Jeljaszewizc, J (ed) Staphylococci and staphylococcal infections. Stuttgart, Gustav Fischer Verlag. pp 9-13.

Marples R R, Richardson J F 1982 Evaluation of a micromethod gallery (API Staph) for the identification of staphylococci and micrococci. Journal of Clinical Pathology 35:650-656.

Marsik F J, Brake S 1982 Species identification and susceptibility to 17 antibiotics of 
coagulase-negative staphylococci isolated from clinical specimens. Journal of Clinical Microbiology 15:640-645.

Oxoid Ltd 1980 Error minimization in susceptibility testing by means of the agar diffusion test. Oxoid Ltd, Basingstoke, Hants.

Price S B, Flournoy D J 1982 Comparison of antimicrobial susceptibility patterns among coagulase-negative staphylococci. Antimicrobial Agents and Chemotherapy 21:436-440.

Richardson J F, Marples R R 1982 Changing resistance to antimicrobial drugs, and resistance typing in clinically significant strains of Staphylococcus epidermidis. Journal of Medical Microbiology 15:475-484.

Sabath L D 1977 Chemical and physical factors influencing methicillin resistance in Staphylococcus aureus and Staphylococcus epidermidis. Journal of Antimicrobial Chemotherapy 3, Suppl C:47-51.

Shannon K, Phillips I 1982 Mechanisms of resistance to aminoglycosides in clinical isolates. Journal of Antimicrobial Chemotherapy 9:91-102.

Weinstein R A, Kabins S A, Nathan C, Sweeney H M, Jaffe H W, Cohen S. 1982 Gentamicin-resistant staphylococci as hospital flora: epidemiology and resistance plasmids. Journal of Infectious Diseases 145:374-382.

Wilkinson B J, Maxwell S, Schaus S M 1980 Classification and characteristics of coagulasenegative, methicillin-resistant staphylococci. Journal of Clinical Microbiology 12:161-166.

Winston D J, Dudnick D V, Chapin M, Ho W G, Gale R P, Martin W J 1983 Coagulase-negative staphylococcal bacteremia in patients receiving immunosuppressive therapy. Archives of Internal Medicine 143:32-36. 